\section{OTH-4 \\ ASSESSING THE EFFECTIVENESS OF CURRENT UK GUIDELINES ON FAMILIAL COLORECTAL CANCER RISK}

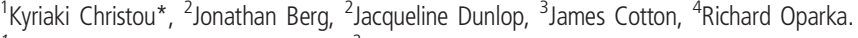
${ }^{1}$ University of Dundee, Dundee, UK; ${ }^{2}$ Department of Clinical Genetics, Ninewells Hospital, Dundee, UK; ${ }^{3}$ Department of Gastroenterology, Ninewells Hospital, Dundee, UK; ${ }^{4}$ Department of Pathology, Ninewells Hospital, Dundee, UK

\subsection{6/gutjnl-2021-BSG.35}

Introduction The British Society of Gastroenterologists (BSG) have produced guidelines for risk stratification and colonoscopic surveillance of people with a family history $(\mathrm{FH})$ and known mutations of colorectal cancer (CRC). To our knowledge, there has been no attempt to validate the empirical BSG criteria for assessing risk in people attending clinical genetics services concerned by their FH of CRC.

Methods FH data was obtained for all unaffected people with a family history of CRC, referred to Tayside clinical genetics from 2000-2009. Risk category according to BSG guidance was assigned de novo (low/population, low-moderate, highmoderate and high). Individuals who went on to develop adenomatous polyps or CRC were identified by record linkage. The risk and the rate of CRC development and adenoma detection were calculated with each group using Relative Risk and Kaplan Meyer Survival Analysis (KMSA) respectively. Analyses involving the high-risk group was conducted both including and excluding mutation carriers (MC).

Results 1120 patients were identified and after exclusion criteria, there were 728 non-polyposis patients (288 low-risk, 316 moderate-risk and 121 high-risk, including $31 \mathrm{MC}$ ) with a total of 5562 patient years of follow-up. Eight invasive CRC developed, 2 in low, 3 in moderate and 3 in high-risk groups ( 2 of those in MC). There were also 65 adenomatous polyps 11,31 and 23 in the respective risk-groups, including 5 in the MC group. There was a significantly increased risk of CRC developing in MC but not in the other risk-groups. KMSA showed no significant difference in the rate of CRC development between the risk-groups. There was a higher risk of detecting polyps in the high-risk group compared to the low risk, especially when less than 50 years old. There was a significantly higher rate of polyp detection in all categories compared to the low-risk group.

Conclusions The mutation group have a significantly higher risk of CRC development, but regular screening appears to reduce this risk. For the rest of the risk-categories, the results show that there was no significant difference in the rate of CRC development when enrolled to a screening programme. Colonoscopic surveillance appears effective in reducing the cancer incidence in high-risk groups below 50 years old, presumably through polyp removal, thereby supporting the current guidance. The study also re-affirms the recommendations that surveillance in medium-risk group is not required below the age of 50 . In this context, BSG guidelines appear to effectively stratify risk for familial CRC and screening in these individuals carries a clinical benefit.

\section{OTH-8 PATIENT REPORTED ANXIETY, BEFORE AND AFTER COLONOSCOPY, DURING THE COVID-19 PANDEMIC}

\footnotetext{
${ }^{1}$ Andrew Prentice*, ${ }^{2}$ Ghalia Kayal, ${ }^{1}$ Sarah Marshall, ${ }^{2}$ Christian von Wagner, ${ }^{2}$ Robert Kerrison. 'St Mark's Bowel Cancer Screening Centre, London, UK; ' University College London, London, UK
}

10.1136/gutjnl-2021-BSG.36
Introduction At the start of the covid-19 pandemic, many invasive diagnostic tests had to be stopped to avoid patients catching covid-19 as a result of attending hospital. Several procedures to reduce COVID transmission have been implemented as services resumed (e.g. COVID testing). Despite this, some patients may still experience what is now called 'COVID anxiety'. The aim of this study was to monitor satisfaction with covid mitigation measures and anxiety among patients attending colonoscopy following an abnormal screening result.

Methods We surveyed patients who were invited for colonoscopy at St Mark's Hospital between July 2020 and May 2021. Data on anxiety and worry were collected, 3 days before and after colonoscopy; scales ranged from 6-24 for general anxiety, 0-20 for COVID-anxiety and 1-4 for bowel cancer worry (with higher scores representing greater anxiety for all three measures). Descriptive statistics were used to assess mean pre- and post-procedure anxiety scores. Inferential statistics were used to test for differences in anxiety, before and after colonoscopy. All analyses were performed using SPSS (Ver 27.0).

Results 205 patients (mean age 67 years; 114 males, 91 females; 121 White, 55, South Asian, 27 any other ethnicity; 142 English first language speakers) completed the pre-procedure questionnaire and were enrolled into the study. 89 (43.4\%) completed the post-procedure questionnaire and were analysed.

Pre- procedure, mean general anxiety, COVID-anxiety, and bowel cancer worry scores were $10.94,0.93$ and 1.95 , respectively. Post-procedure, the general anxiety, COVID-anxiety and bowel cancer worry scores were 8.92, 1.03 and 2.11, respectively. The change in general anxiety $(-2.02)$ was statistically significant $(\mathrm{p}=0.015)$, while the changes in COVID anxiety $(+0.1)$ and bowel cancer worry $(+0.16)$ were not (both ps<0.05).

Significant reductions in general anxiety were observed for women (pre- and post-procedure general anxiety scores were: 13.05 vs. 8.33, respectively; $\mathrm{p}<0.001$ ), White British adults (pre- and post-procedure general anxiety scores were: 11.47 and 8.90, respectively; $\mathrm{p}<0.001$ ) and adults whose first language was English (pre- and post-procedure general anxiety scores were: 11.49 and 9.08, respectively; $p<0.001)$. The majority of patients were highly satisfied with the covid-measures put in place. There was no association between satisfaction and changes in COVID Anxiety $(p<0.05)$.

Conclusions COVID anxiety was low among people who attended colonoscopy. General anxiety, however, was moderate, although it was reduced following the procedure. General anxiety was not reduced for some groups, including those whose first language is not English, highlighting the need for further research into factors affecting experience in these groups.

\section{Education \& training}

\section{OTU-15 BSG TRAINEES SECTION SURVEY 2020 - THE IMPACT OF COVID-19 ON UK GASTROENTEROLOGY TRAINING}

Elizabeth Ratcliffe, Rebecca Harris, Suneil Raju*, Charlotte Cook, Phil Harvey. British Society of Gastroenterology Trainees section, London, UK

10.1136/gutjnl-2021-BSG.37 
Introduction The impact of the COVID-19 pandemic has disrupted training during the initial peak and partial recovery. Gastroenterology higher speciality training (HST) is reaching an inflection point with a reduction from five years to four. The potential compound impact is a source of concern for HSTs. The BSG Trainees Section biennial survey 2020 aimed to delineate the impact of COVID-19 and opinions on changes to training.

Methods An electronic survey allowing for anonymised responses at the point of completion was distributed to all gastroenterology HSTs over a three month period from September to November 2020.

Results In total, 349 trainees completed the survey (response rate of $51 \%$ of 687 HSTs) with representation across all regions. $89 \%(307 / 344)$ of responders were full time trainees and $39 \%(136 / 349)$ female. There was a reduction in access to clinics; 48.4\%(169/349) reported due to general internal medicine (GIM) duties and $26.6 \%$ (93/349) due to reduction in available clinics. Reduction in experience in gastroenterology referrals were also mainly limited by GIM commitments (42.4\%) (148/349). No endoscopy training occurred for 88.5 $(170 / 192) \%$ of trainees at the peak. Recovery of training lists was reported by $67.3(175 / 260) \%$ of responders in late summer yet $20.6(72 / 349) \%$ reported training lists were still 'not allowed' in their trust. 71.0 (206/290)\% of responders reported their time was predominantly taken by GIM, with $42.1(110 / 261) \%$ considering the need to extend their CCT date to compensate for this. $49.0 \%(128 / 261)$ of respondents were considering time out of programme or fellowships and $28.3(74 / 261) \%$ considering it in the future. The majority of responders reported virtual or online teaching was provided during the peak of the pandemic.

Considering future training; 96.8\%(245/253) of respondents stated gastroenterology HSTs should have 1 year experience on a GI bleed rota, however only $21.3 \quad(55 / 258) \%$ reported having experience of this formally during training. $68.8 \%(174 /$ 253) of responders supported the idea of blocks of GIM training during HST to protect gastroenterology training. The majority of trainees $(84.2 \%)(213 / 253)$ reported they would not feel ready to be a consultant after 4 years of HST. 46.7 $(122 / 261) \%$ of responders would stop GIM training if given the opportunity.

Conclusions In all aspects of gastroenterology training surveyed, more than half of training time was lost during the pandemic. This included training beyond endoscopy to other aspects of GI work including clinics and referrals. This is mirrored in anticipated concerns about completion of training and the perceived future competence as a consultant at the end of a 4 year higher training programme. Work is now required to ensure training trajectories are restored following the COVID-19 pandemic.

\section{OTU-16 DEVELOPMENT OF THE TEAM-ENTS (TEAMWORK IN ENDOSCOPY ASSESSMENT MODULE FOR ENDOSCOPIC NON-TECHNICAL SKILLS) FRAMEWORK}

\footnotetext{
${ }^{1,2,3}$ Srivathsan Ravindran* ${ }^{1}$ Chris Healey, ${ }^{1}$ Mark Coleman, ${ }^{3}$ Hutan Ashrafian,

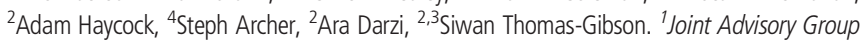
On GI Endoscopy (JAG), London, UK; ${ }^{2}$ Wolfson Unit for Endoscopy, St Mark's Hospital, London, UK; ${ }^{3}$ Imperial College London, London, UK; ${ }^{4}$ Department of Public Health and Primary Care, Cambridge University, Cambridge, UK
}

Introduction Teamworking is crucial to team performance and has been demonstrated to improve the quality and safety of care. Non-technical Skills (NTS) are integral to teamworking and NTS frameworks have been used to support training and assessment in healthcare. The aim of this study was to develop a novel team-based NTS framework in endoscopy (TEAM-ENTS).

Methods A qualitative mixed-methods study was conducted to ascertain the core NTS relevant to endoscopy teams. This was undertaken in two phases: 1) a detailed literature review of team-based NTS frameworks and, 2) an interview study of endoscopists and endoscopy nurses utilising Cognitive Task Analysis (CTA). Transcribed interviews were analysed by two researchers using framework analysis. A codebook of relevant skills and behaviours was created which formed the basis for the TEAM-ENTS framework.

Results 1162 articles were screened, identifying 36 articles which described 14 team-based NTS frameworks. In total, 13 semi-structured interviews were completed involving 7 endoscopists and 6 nurses. The mean number of years in role was 15.2 and mean lifetime procedure count was 9571. 88 codes were identified from transcribed interviews. These fell into 5 major categories: 1) Communication, 2) Planning, decision-making and problem-solving, 3) Leadership and coordination, 4) Situation awareness and 5) Teamwork, cooperation and support. A hierarchical framework was created of overarching categories further defined by individual elements (Table 1). Elements are characterised by behavioural descriptors informed by the literature review. The final framework consists of 5 categories, 17 elements and 58 behavioural descriptors.

\begin{tabular}{ll} 
Abstract OTU-16 Table 1 & \\
\hline CATEGORY & ELEMENT \\
\hline COMMUNICATION & 1. Information exchange \\
& 2. Maintaining open dialogue \\
PLANNING, DECISION-MAKING \& PROBLEM- & 4. Patient communication \\
SOLVING & 5. Joint decision-making \\
& 6. Reviewing outcomes \\
LEADERSHIP \& COORDINATION & 7. Control and responsibility \\
& 8. Role delegation \\
SITUATION AWARENESS & 9. Team adaptation \\
& 10. Team diagnosis \\
11. Problem recognition and \\
focus \\
12. Shared understanding \\
13. Anticipating events \\
14. Teambuilding and trust \\
15. Cohesion \\
16. Support \\
17. Assertion
\end{tabular}

Conclusions The TEAM-ENTS framework contains the core NTS relevant to endoscopy teams. Further work is underway to refine the tool through an online Delphi study followed by an observational validation study. 